\title{
Study on isolates of acute meningitis in a tertiary care centre in Assam
}

\section{Gitali Bhagawati, Dipa Barkataki, N. K. Hazarika}

Department of Microbiology, Guwahati Medical College and Hospital, Guwahati, Assam, India

Address for the Correspondence: Dr. Gitali Bhagawati, House no. 320, Sainik Vihar, Pitampura, Delhi - 110 034, India. E-mail: gitalibhagawati32@gmail.

com

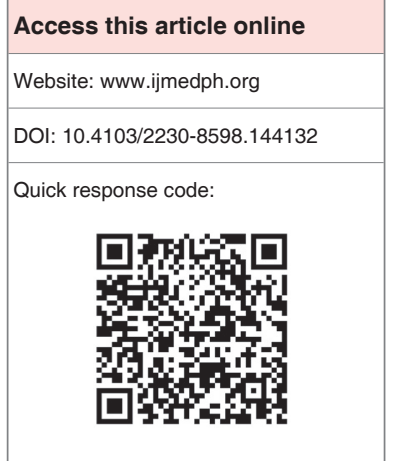

Background: Meningitis is an inflammatory affection of the membranes surrounding the brain and spinal cord, which occurs as either a primary disease or secondarily to disease in some other part of the body. The epidemiological trend of acute meningitis varies with time and geography. Objective: To isolate the various agents of acute meningitis in all age group patients and to know the antimicrobial susceptibility pattern of bacterial isolates. Materials and Mathods: In this prospective study, a total of 316 cerebrospinal fluid (CSF) specimens were collected from patients showing signs and symptoms of acute meningitis and processed by standard microbiological methods in a tertiary care hospital in Guwahati, Assam over a period of one year, from August 2009 to July 2010. Results: Out of 316 CSF samples, bacterial and fungal culture positivity rate was found to be $16.13 \%$. The most common bacterial isolate was Staphylococcus aureus, $29.41 \%$. Isolation rate of Cryptococcus neoformans was $8 \%$. All the Gram positive isolates were $100 \%$ sensitive to linezolid and vancomycin, whereas Gram negative isolates were $92 \%$ sensitive to polymyxin $\mathrm{B}$. Conclusion: This study gives an idea about the changing trend of acute meningitis along with the changing in vitro antimicrobial susceptibility pattern of the bacterial isolates, which can help the clinicians to formulate the initial empiric therapy for patients of acute meningitis.

Key words: Acute meningitis, MRSA, sensitivity pattern

\section{INTRODUCTION}

Meningitis is an inflammatory affection of the membranes surrounding the brain and spinal cord, which occurs as either a primary disease or secondarily to disease in some other part of the body. It is associated with significant morbidity and mortality in all parts of the world, particularly, in pediatric age group. ${ }^{[1]}$ Causative agents of acute meningitis can be viral, bacterial or fungal pathogens. Among these, bacterial meningitis can be quite severe and may result in brain damage, hearing loss or learning disability and death. ${ }^{[2]}$

Among the bacterial agents causing meningitis, the most common agents responsible for communityacquired bacterial meningitis are Streptococcus pneumoniae ( $50 \%$ ), Neisseria meningitidis ( $25 \%$ ), Group B Streptococcus $(15 \%)$ and Listeria monocytogens ( 10\%). Hemophilus influenzae now accounts for less than $10 \%$ of cases of bacterial meningitis. The epidemiology of bacterial meningitis has changed significantly in recent years, reflecting a dramatic decline in the incidence of meningitis caused by Hemophilus influenzae and Neisseria meningitidis, following the introduction and increasingly widespread use of vaccines for both these organisms. Enteric Gram negative bacilli are increasingly common cause of meningitis in individual with chronic and debilitating diseases like diabetes, cirrhosis or alcoholism and in those with chronic urinary tract infection. Staphylococcus aureus and coagulasenegative staphylococcus are important causes of meningitis that follow invasion of neurosurgical procedures. ${ }^{[3]}$

In most studies, besides conventional pathogens other bacteria like Klebsiella species, Escherichia coli, Pseudomonas species, Acinetobacter species, Citrobacter species, Enterococcus species etc. have been isolated from admitted patients.

In partially treated meningitis, CSF may become clear with predominant lymphocytes; culture may also be sterile. ${ }^{[4]}$ 
Cryptococcal meningitis caused by environmental fungus Cryptococcus neoformans, is a common opportunistic infection in human immunodeficiency (HIV)-infected individuals, particularly in Southeast Asia, Southern and East Africa. ${ }^{[5,6]}$ It usually infects the HIV-infected patients with CD 4 count $<100$ cells $/ \mu$ l. These patients present with features of acute, sub-acute or chronic meningitis or meningoencephalitis. ${ }^{[6]}$

\section{MATERIALS AND METHODS}

A total of 316 CSF samples were tested in the Department of Microbiology in a tertiary care hospital in Guwahati, Assam over a period a period of one year, from August 2009 to July 2010.

CSF samples were collected as a part of the routine clinical management from patients with signs and symptoms of acute meningitis and were admitted in different wards of the hospital that include: Medicine wards, pediatric wards, intensive care units (ICU), neonatal intensive care units (NICU) etc.

The CSF samples were collected in sterile containers by attending physicians and delivered to the Microbiology laboratory as soon as possible. CSF of $2-3 \mathrm{ml}$ was transferred to a sterile centrifuge tube and was centrifuged at $3000 \mathrm{rpm}$ for 5 minutes. The supernatant part was transferred to a clean container for chemical examination. ${ }^{[7]}$

The CSF deposits were seeded on to blood agar, chocolate agar, and chocolate agar medium with vitamino growth supplements, modified (twin pack) (HiMedia Laboratories, FD215), Mac Conkey agar and glucose broth. The Mac Conkey agar and glucose broth were incubated aerobically while blood agar, chocolate agar, chocolate agar medium with supplements were incubated in the presence of $5-10 \%$ carbon dioxide in candle jar at $37^{\circ} \mathrm{C}$ for 24 hours. The plates and broth were examined after overnight incubation and if there was no growth the plates were re-incubated for further 24 hours and examined. The broth was sub-cultured on the above solid media and examined.

Organisms were identified by standard microbiological methods, which included colony morphology, as well as staining, and biochemical tests. ${ }^{[1,8,9]}$

The criteria for diagnosis of Cryptococcus neoformans were based on positive India ink preparation of CSF, culture in Sabouraud dextrose agar and Bird seed agar, assimilation test, biochemical test and latex agglutination test. ${ }^{[10]}$

The criteria for diagnosis of Candida albicans were based on $\mathrm{KOH}$ mount of CSF, culture in Sabouraud dextrose agar and CHROM agar, assimilation reaction and germ tube test. ${ }^{[1]}$

Antibiotic sensitivity test was conducted on pure culture isolates employing the disc diffusion method for the commonly used antibiotics per disc: Penicillin G (P, $10 \mu \mathrm{g})$, gentamicin (G, 10 $\mu \mathrm{g})$, amikacin $(\mathrm{AK}, 30 \mu \mathrm{g})$ erythromycin $(\mathrm{E}, 5 \mu \mathrm{g})$ ), vancomycin

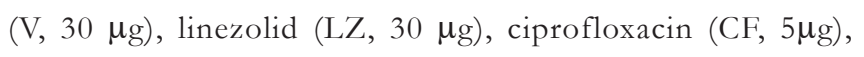
amoxicillin-clavulanic acid (AC, $10 \mu \mathrm{g}$ ), piperacillin- tazobactum (PZ, 100/10 $\mu \mathrm{g}$ ), cefotaxime (CE, $30 \mu \mathrm{g})$, ceftriaxone (CI, $30 \mu \mathrm{g})$, cefoperazone (CS, $75 \mu \mathrm{g})$, ceftazidime (CZ, $30 \mu \mathrm{g})$, cefoxitin (CN, $30 \mu \mathrm{g})$ and polymyxin $\mathrm{B}(\mathrm{Pb}, 300 \mathrm{U})$, (HiMedia). The diameters of growth inhibition around the discs were measured and interpreted as sensitive, intermediate or resistant as per the Clinical and Laboratory Standards Institute (CLSI) guideline 2010. ${ }^{[12]}$ Reference strains used as controls were E. coli ATCC 25922 and S. aureus ATCC 25923.

\section{RESULTS}

A total of 316 CSF samples were investigated during the study period from August 2009 to July 2010.

In this study, out of total 316 CSF samples, 163 (51.58\%) samples were collected from patients of the age group 0-10 years, followed by $41(12.97 \%)$ in the age group of $11-20$ years. Only one sample $(0.32 \%)$ was collected above the age of 70 years.

Out of the total 316 cases, $212(67.09 \%)$ were males and 104 $(32.91 \%)$ were females; the male to female ratio is $2: 1$.

Fever $(92.72 \%)$, altered mental status $(74.36 \%)$, headache $(61.71 \%)$ and convulsion (57.6\%) were the most common clinical manifestations of meningitis among the patients in the present study. Frequencies of clinical manifestations of patients are shown in Table 1.

Out of 316 CSF samples, bacterial as well as fungal pathogens were isolated from 51 samples showing an isolation rate of $16.14 \%$. The patients showing CSF culture positivity also had some predisposing conditions, with altered CSF biochemical levels as well as pathological counts.

Out of total $51(16.14 \%)$ culture positive samples, $44(86.27 \%)$ samples were positive for bacteria whereas $7(13.72 \%)$ samples were positive for fungus. No sample was found to be positive for Mycobacteria.

\begin{tabular}{lc}
$\begin{array}{l}\text { Table 1: Clinical manifestation of acute meningitis } \\
\text { in } 316 \text { cases }\end{array}$ \\
\hline Clinical features & No. of cases (\%) \\
\hline Fever & $293(92.72)$ \\
Altered mental status & $235(74.36)$ \\
Headache & $195(61.71)$ \\
Convulsion & $182(57.59)$ \\
Vomiting & $178(56.33)$ \\
Irritability & $167(52.85)$ \\
Neck rigidity & $138(43.67)$ \\
Kernig's sign & $110(34.81)$ \\
Bulging fontanel & $51(16.14)$ \\
Refusal to feed & $49(15.51)$ \\
Skin rash & $5(1.58)$ \\
\hline
\end{tabular}




\section{DISCUSSION}

There is a need for periodic review of acute meningitis worldwide, since the pathogens responsible for infection vary with time, geography and patient's age. Increase in awareness, availability of vaccines may also reflect a change in the epidemiological pattern of these pathogens. The etiological agents of community-acquired meningitis may differ from hospital- acquired meningitis. Delay in diagnosis and initiation of treatment can result in poor outcome of the disease. ${ }^{[13]}$ Thus, the data presented in this study can provide information of immediate public health importance to clinicians on the selection of antimicrobial agents for the treatment of patients suffering from acute meningitis.

Out of total suspected cases of meningitis, 163 (51.58\%) CSF samples were collected from patients of the age group 0-10 years, followed by $41(12.97 \%)$ in the age group of 11-20 years. Only one sample $(0.32 \%)$ was collected above the age of 70 years. This was similar to the study of Emele $\mathrm{e}^{[14]}$ where $68 \%$ cases were in the age group 1-9 years and this, however contrasted with Mani et al. ${ }^{[15]}$ where $86.8 \%$ were adults and $13.2 \%$ were below the age of 12 years.

Out of the total 316 cases, $212(67.09 \%)$ were males and 104 $(32.91 \%)$ were females; the male to female ratio is $2: 1$. Similar observations were made by Mani et al. ${ }^{[15]}$ [male 293 (76.1\%) and female $92(23.9 \%)$ ].

Fever $(92.72 \%)$, altered mental status $(74.36 \%)$, headache $(61.71 \%)$ and convulsion $(57.6 \%)$ were the most common clinical manifestations of meningitis among the patients in the present study [Table 1]. Similarly, fever was observed by Chinchankar et al. ${ }^{[16]}$ in 52 cases $(96 \%)$, on the contrary, Abdul Khaliq ${ }^{[17]}$ reported fever only in $7(33.33 \%)$ cases.

Out of total 316 cases, predisposing conditions were recorded in $143(45.25 \%)$ patients. The most common predisposing condition was diabetes 45 (14.24\%), followed by pre-term delivery $36(11.39 \%)$ among neonates. HIV/AIDS were recorded in 17 $(5.38 \%)$ patients [Figure 1]. Similarly, Tang et al. ${ }^{[18]}$ mentioned various pre-disposing conditions, e.g. diabetes mellitus, head injury and/or neurosurgical procedure, and malignancy in $71.8 \%$ of patients.

The isolation rate of $16.14 \%$ found in the present study is in agreement with a various studies by different authors. ${ }^{[19,20]}$ Even though all febrile patients with signs and symptoms of acute meningitis have underwent lumbar puncture to rule out acute meningitis, the bacterial isolation rate was found to be low. The predominant organism isolated from CSF culture was found to be Staphylococcus aureus 15 (29.41\%) [Table 2]. Higher prevalence of meningitis due to Staphylococcus aureus may be due to predisposing factors as well as co-morbidities associated with the patient. Similar finding was observed by Wen-Neng Chang $(23.8 \%)^{[21]}$, Rasoul et al. (13.1\%). ${ }^{[2]}$ However, the study was dissimilar to the findings observed by Mani et al $(1.8 \%) \cdot{ }^{[15]}$

Among the Gram negative isolates, Klebsiella sp. was found to be the most common isolate, 8 (15.7\%). Other Gram negative bacilli were Escherichia coli 6 (11.76\%), Acinetobacter sp. 4 (7.84\%), Pseudomonas sp. 3 (5.88\%) and Citrobacter sp. 2 (4\%). The change in the epidemiological trend of isolated organisms in meningitis is due to hospital-acquired infection associated with increasing immunocompromised status of

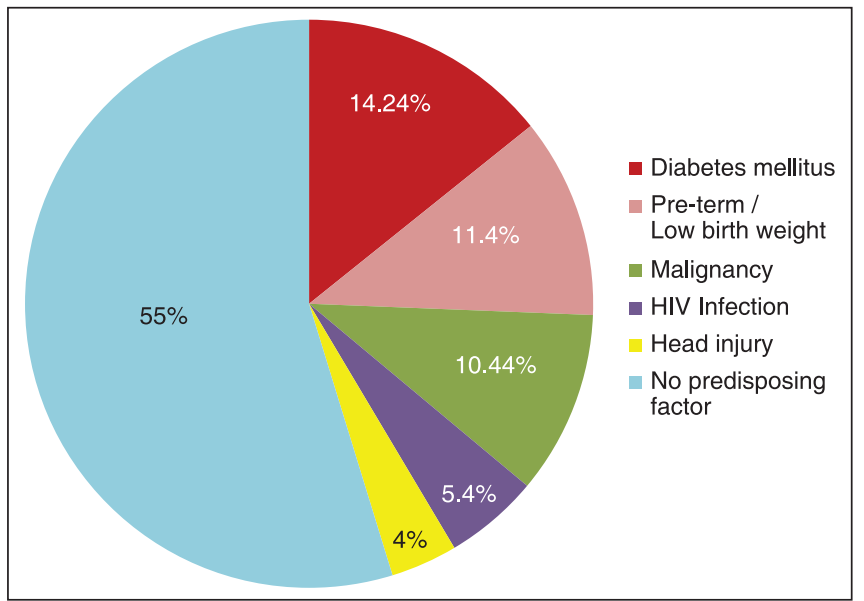

Figure 1: Predisposing conditions associated with 143 (45.25\%) cases of acute meningitis

\begin{tabular}{|c|c|c|c|c|c|c|c|}
\hline Isolated organisms & No. (\%) & Male & Female & $\begin{array}{c}\text { newborn to } 3 \\
\text { months }\end{array}$ & $\begin{array}{c}>3 \text { months to } 10 \\
\text { years }\end{array}$ & $11-45$ years & $>45$ years \\
\hline Staphylococcus aureus & $15(29.41)$ & 12 & 3 & 3 & 6 & 3 & 3 \\
\hline Klebsiella sp. & $8(15.7)$ & 7 & 1 & 1 & 4 & 1 & 2 \\
\hline Escherichia coli & $6(11.76)$ & 3 & 3 & 3 & 2 & 1 & 0 \\
\hline Acinetobacter sp. & $4(7.84)$ & 4 & 0 & 0 & 0 & 2 & 2 \\
\hline Cryptococcus neoformans & $4(7.84)$ & 3 & 1 & 0 & 0 & 2 & 2 \\
\hline Pseudomonas sp. & $3(5.88)$ & 1 & 2 & 0 & 0 & 3 & 0 \\
\hline Candida albicans & $3(5.88)$ & 3 & 0 & 2 & 0 & 0 & 1 \\
\hline Listeria sp. & $2(4)$ & 2 & 0 & 1 & 1 & 0 & 0 \\
\hline Niesseria meningitidis & $2(4)$ & 1 & 1 & 0 & 1 & 0 & 1 \\
\hline Streptococcus pneumoniae & $2(4)$ & 1 & 1 & 0 & 0 & 1 & 1 \\
\hline Citrobacter sp. & $2(4)$ & 2 & 0 & 2 & 0 & 0 & 0 \\
\hline Total & $51(100)$ & 39 & 12 & 12 & 14 & 13 & 12 \\
\hline
\end{tabular}


the patients like diabetes, HIV/AIDS, pre-term delivery, malignancy etc. Prolonged hospital stay, improper hand hygiene practice by the care givers, inappropriate patient-health care workers ratio were some other causes of hospital-acquired meningitis by these organisms.

Isolation rate of Streptococcus pneumoniae and Neisseria meningitidis were less in the study. Hemophilus influenzae have not been isolated from CSF in this study. This may be because of fastidious nature of the organisms, vaccine implementation against these organisms or antibiotic treatment prior to lumber puncture. The CSF becomes sterilized within 4 hours of parenteral antibiotic treatment in case of pneumococcal meningitis. ${ }^{[23]}$ The lowered incidence of meningococcal meningitis in the present study is probably due to the occurrence of meningitis during the interepidemic period.

Among the fungal isolates Cryptococcus neoformans 4 (7.84\%) predominated over Candida albicans 3(5.88\%). The single predisposing factor for Cryptococcus neoformans meningitis was found to be AIDS. Out of 4 patients, $3(75 \%)$ responded to amphotericin B therapy whereas the other patient died of Cryptococcamea. Candida albicans were isolated from two pre-term neonates and one 49-year male patient with leukemia. All of them responded to fluconazole.

Among the 15 isolates of Staphylococcus aureus, 80\% was found to be sensitive to amoxycillin-clavulanic acid followed by amikacin 60\% each. Among the total 15 isolates of Staphylococcus aureus, 3 (20\%) were methicillin-resistant S. aureus (MRSA). Inappropriate as well as excessive use of broadspectrum antibiotics and prolonged hospital stay are the other causes of emergence of MRSAs. Garcia-Arias et al. ${ }^{[24]}$ isolated S. aureus in $3 \%$, among which MRSAs was $47.6 \%$ which is almost similar to the observation of this study. MRSAs isolated in the previous study of the same institution were $14 \%{ }^{[25]}$ Higher prevalence of meningitis due to $S$. aureus, in the present study, may be due to predisposing factors as well as co-morbidities associated with the patient. Inappropriate and injudicious use of broadspectrum antibiotics, prolonged hospital stay, overcrowding of the hospital environment, inadequate practice of hand hygiene, non-isolation of MRSA patients were the causes behind the emergence of higher prevalence of MRSAs.

However, all the Gram-positive isolates of CSF are found to be sensitive to newer antibiotics like linezolid and vancomycin [Table 3].

The in vitro susceptibility tests of the bacterial isolates revealed that polymyxin B 92\% was the most effective antimicrobial agent against the entire spectrum of the Gram-negative bacilli. It was followed by imipenem $88 \%$ and piperacillin-tazobactum $64 \%$. The sensitivity of each of ciprofloxacin and amikacin was $56 \%$, followed by amoxycillin-clavulanic acid $52 \%$ [Table 4]. Sonavane et al. ${ }^{[13]}$ found 2 isolates of multi-drug resistant (MDR) Acinetobacter spp., as they were resistant to higher antibiotic like imipenem.

\section{CONCLUSION}

Increasing resistance to empirically used antimicrobials in meningitis especially to cephalosporins (cefoperazone, ceftriaxone, cefotaxime, ceftazidime etc.) is an alarming condition. Resistance to newer drugs like imipenem is another alarming condition for the clinicians.

For public health concern, the etiological change in the trend of meningitis and the risk factors associated with meningitis should be reviewed from time-to-time. Along with these surveys, continued surveillance for resistance characteristics among the organisms is necessary. This study gives a clear idea about the prevalence of organisms related with meningitis in this part of the country along with the changing in vitro antimicrobial susceptibility pattern of the bacterial isolates. Producing updated information on local causative pathogens and their antibiotic sensitivity pattern can help the clinicians to formulate the initial empiric therapy. Prevention of the emergence of antimicrobial resistant bugs by promotion of judicious use of antibiotics can provide a long-term solution.

\begin{tabular}{lccccccccc}
\hline $\begin{array}{l}\text { Table 3: Antibiotic sensitivity pattern of Gram-positive bacteria isolated from CSF } \\
\text { Antibiotics }\end{array}$ & P\% & AC\% & VA\% & LZ\% & G\% & AK\% & E\% & CF\% & Cl\% \\
\hline Isolates & & & & & & & & & \\
S. aureus (15) & 26.6 & 80 & 100 & 100 & 53.3 & 60 & 46.6 & 53.33 & 13.33 \\
S. pneumoniae (2) & 50 & 100 & 100 & 100 & 100 & 100 & 100 & 100 & 50 \\
Listeria sp. (2) & nil & 100 & 100 & 100 & 50 & 50 & 50 & 50 & 50 \\
\hline
\end{tabular}

\begin{tabular}{|c|c|c|c|c|c|c|c|c|c|c|}
\hline Antibiotics & $\mathbf{P} \%$ & $\mathrm{AC} \%$ & PT\% & AK\% & CF\% & $\mathrm{Cl} \%$ & CE\% & $\mathrm{CS} \%$ & $\mathrm{CZ} \%$ & $1 \%$ \\
\hline \multicolumn{11}{|l|}{ Isolates } \\
\hline Klebsiella sp. (8) & nil & 75 & 87.5 & 62.5 & 75 & 25 & 37.5 & 12.5 & 37.5 & 87.5 \\
\hline E. coli $(6)$ & nil & 66.6 & 83.3 & 83.3 & 66.6 & 16.6 & 33.3 & 16.6 & 50 & 100 \\
\hline Acinetobacter sp. (4) & nil & 25 & 50 & 25 & 25 & nil & 25 & nil & 25 & 75 \\
\hline Pseudomonas sp. (3) & nil & nil & 33.3 & 33.3 & 66.6 & nil & 33.3 & nil & 66.6 & 100 \\
\hline Neisseria meningitidis (2) & 50 & 50 & nil & 50 & 50 & 50 & 50 & nil & 50 & 50 \\
\hline Citrobacter sp. (2) & nil & 50 & 50 & 50 & nil & nil & 50 & nil & 50 & 100 \\
\hline
\end{tabular}




\section{ACKNOWLEDGMENT}

We, the authors are grateful to the Principal cum Chief Superintendent of the tertiary care hospital, Assam for allowing us to do the study.

\section{REFERENCES}

1. Smith GR. Topley and Wilson's Principles of Bacteriology, Virology and Immunology 7th edition, 1984;3:369.

2. Richard EB, et al. Nelson textbook of pediatrics. New York: W. B. Saunders Company. 2000;16:707.

3. Roos KL, Tyler KL. Harrison's principle of internal medicine. 2004;16:2471-7.

4. Ghai OP, Gupta P, Paul VK. Central nervous system. Essent Pediatr 2001;5:393.

5. Bicanic T, Harrison TS. Cryptococcal meningitis. Br Med Bull 2005;72: 99-118.

6. Kumar S, Wanchu A, Chakrabarti A, Sharma A, Bambery P, Singh S. Cryptococcal meningitis in HIV infected: Experience from a North Indian tertiary center. Neurol India 2008;56:444-9.

7. Blood Safety and Clinical Technology. Guidelines on Standard Operating Procedures for Microbiology. World Health Organization: Available from: http://searo.who.int/EN/Section10/Section17/Section53/ Section482 1794.htm [Last accessed on 2007 Dec].

8. Brooks GF, Carroll KC, Butel JS, Morse SA, Jawetz, Melnick and Adelberg's Medical Microbiology, McGrawHill Lange; 2004;24:174-94.

9. Vandepitte J, Verhaegen J, Engbaek K, Rohner P, Piot P, Heuck CC. Basic laboratory procedures in clinical bacteriology, Geneva, Switzerland. World Health Organ 2003;2:25-9.

10. Chander J. Cryptococcosis. Textbook of Medical Mycology, $3^{\text {rd }}$ edition; Mehta publication; 2009;291-14.

11. Chander J. Cryptococcosis. Textbook of Medical Mycology, $3^{\text {rd }}$ edition; Mehta publication; 2009;266-90.

12. Performance standards for antimicrobial susceptibility testing; twentieth information supplements Clinical and Laboratory Standard Institute Document M100-S20 2010;30:108-9.

13. Sonavane A, Baradhar VP, Mathur M. Bacteriological profile of pyogenic meningitis in adults. Bombay Hosp J 2008;50:3.

14. Emele FE. Etiologic spectrum and pattern of antimicrobial drug susceptibility in bacterial meningitis in Sokoto, Nigeria. Acta Paediatr 2000;89:942-6.

15. Mani R, Pradhan S, Nagarathna S, Wasiulla R, Chandramuki A. Bacteriological profile of community acquired acute bacterial meningitis: A ten-year retrospective study in a tertiary neuro-care centre in South India. Indian J Med Microbiol 2007;25:108-14.

16. Chinchankar N, Mane M, Bhave S, Bapat S, Bavdekar A, Pandit A, et al. Diagnosis and outcome of acute bacterial meningitis in early childhood. Indian Pediatr 2002;39:914-21.

17. Sallam AK. Etiology and presentation of acute bacterial meningitis in children at Al-Thawrah Hospital, Sana'a, Yemen. J Ayub Med Coll Abottabad 2004;16:40-3.

18. Tang LM, Chen ST, Hsu WC, Chen CM. Klebsiella meningitis in Taiwan: An overview. Epidemiol Infect 1997;119:135-42.

19. Matee MI, Matre R. Pathogenic isolates in meningitis patients in Dar Es Salaam, Tanzania. East Afr Med J 2001;78:458-60.

20. Chakrabarti P, Das BK, Kapil A. Application of $16 \mathrm{~S}$ rDNA based seminested PCR for diagnosis of acute bacterial meningitis. Indian J Med Res 2009;129:182-8.

21. Chang WN, Lu CH. Diagnosis and management of adult bacterial meningitis. Acta Neurol Taiwan 2009;18:3-13.

22. Rasoul YM, Sayyed HH, Manije B. Bacterial agents of meningitis in children and detection of their antibiotic resistance patterns in Hamadan, Western Iran. Pak J Biol Sci 2006;9:1293-8.

23. Keith AV. Cartwright. Bacterial Meningitis. Topley and Wilson's microbiology and microbial infections. Vol. 10. Italy: Hodder Arnold; 2005 p. 555-7.

24. Garcia-Arias V, Gomez-Rodriguer JA, Sanchez Somolionos M, Alanso P, Garcia Lechuz JM, Palaez T, et al. S. Aureus Isolates from Cerebrospinal Fluid: A Microbiological and Clinical Study. Interscience Conference on Antimicrobial Agents and Chemotherapy 2003; 43rd: Chicago, III.

25. Rasul ES, Deka PK. Study of bacterial meningitis in Assam. Indian Med Gaz 1998;CXXXII:151-6.

How to cite this article: Bhagawati G, Barkataki D, Hazarika NK. Study on isolates of acute meningitis in a tertiary care centre in Assam. Int J Med Public Health 2014;4:446-50.

Source of Support: Nil, Conflict of Interest: None declared. 